\title{
Flebótomos (Diptera: Psychodidae) del departamento de Guaviare, Colombia, con nuevos registros para el país
}

\author{
Olga Lucía Cabrera ${ }^{1}$, Laureano Mosquera², Erika Santamaría¹, Cristina Ferro ${ }^{1}$ \\ ${ }^{1}$ Grupo de Entomología, Subdirección de Investigación, Instituto Nacional de Salud, Bogotá, D.C., Colombia \\ 2 Secretaría de Salud del Guaviare, San José del Guaviare, Colombia
}

Introducción. El registro de casos de leishmaniasis en el departamento de Guaviare evidenció la ausencia de estudios entomológicos dirigidos a identificar las especies de Lutzomyia de esa región del país.

Objetivo. Determinar las especies del género Lutzomyia en los cuatro municipios del departamento del Guaviare y señalar por antecedentes vectoriales las especies con posible compromiso en la transmisión de la leishmaniasis.

Materiales y métodos. El muestreo se realizó con trampas CDC colgadas entre 1,5 m y $9 \mathrm{~m}$ de altura. Además, se recolectaron flebótomos con trampa Shannon y en sitios de reposo.

Resultados. Se recolectaron flebótomos pertenecientes a 37 especies; 35 de ellas se registran por primera vez para el departamento del Guaviare y cuatro para el país: L. begonae, L. campbelli, $L$. sericea y $L$. nematoducta. Las especies más abundantes fueron $L$. hirsuta $24,3 \%$ (148/610), $L$. yuilli $15,2 \%$ (93/610) y L. davisi 10,3\% (63/610), seguidas por L. fartigi, L. carrerai, L. antunesi, $L$. flaviscutellata y L. olmeca bicolor.

Conclusión. Se identificaron siete especies de Lutzomyia como posibles vectores de leishmaniasis.

Palabras clave: Lutzomyia, leishmaniasis cutánea, biodiversidad, ecosistema amazónico, Colombia.

Sand flies (Diptera: Psychodidae) of Guaviare Province, Colombia, with 4 new records for the country

Introduction. Although cases of leishmaniasis have been reported in the province of Guaviare, Colombia, no entomological studies were included to identify the Lutzomyia sand fly vector species in that area.

Objective. Lutzomyia species were identified from four townships of Guaviare. Probable vectors were named based on those species involved in transmission in other areas.

Materials and methods. Sampling was undertaken with CDC light traps suspended at heights between $1.5 \mathrm{~m}$ and $9 \mathrm{~m}$. Additional sand flies were collected with Shannon traps and by aspiration of adult flies from daytime resting sites.

Results. Sand flies belonging to 37 different species were collected. 35 of them were recorded for the first time in Guaviare Province. Four species were new records for Colombia: Lutzomyia begonae, L. campbelli, L. sericea and L. nematoducta. The most abundant species were $L$. hirsuta $24.3 \%$ (148/610), L. yuilli 15.2\% (93/610), L. davisi 10.3\% (63/610), followed by L. fartigi, L. carrerai, L. antunesi, L. flaviscutellata and L. olmeca bicolor.

Conclusion. Seven of these species of have been associated previously with endemic or epidemic transmission of leishmaniasis.

Key words: Lutzomyia; leishmaniasis, cutaneous; biodiversity, Amazonian ecosystem, Colombia. 
Los flebótomos hembras son hematófagos, conocidos como los únicos vectores de leishmaniasis y de otras enfermedades típicas de áreas tropicales y subtropicales en el mundo; su pequeño tamaño $(2,5$ a $3,5 \mathrm{~mm})$ les permite pasar en algunas ocasiones inadvertidos.

La diversidad de especies es muy grande; se han descrito más de 800 especies en el mundo (1), para las cuales los taxónomos han propuesto varios esquemas de clasificación. El más utilizado es el de Lewis y colaboradores (2), el cual reconoce tres géneros: Lutzomyia, Brumptomyia y Warileya para el Nuevo Mundo (Norteamérica, Centroamérica y Suramérica), y Phlebotomus y Sergentomyia para el viejo continente (Europa, África y Asia). Las especies con importancia médica pertenecen a los géneros Lutzomyia y Phlebotomus. El mayor aporte al conocimiento de las especies del género Lutzomyia fue realizado por Young y Duncan (1), quienes en un volumen reunieron, para las 379 especies conocidas, toda la información relacionada, mapas de distribución, dibujos y claves para la identificación a nivel de especie $(3,4)$.

En Colombia las primeras especies registradas fueron L. panamensis y L. squamiventris (error de identificación, se trataba de una nueva especie que correspondió a $L$. fairtigi) recolectadas en las veredas de Caibe y Sardinata, municipio de Restrepo en el departamento del Meta (Orinoquia), entre diciembre de 1934 y marzo de 1935, por el entomólogo brasilero P. Antunes (5). A partir de esa fecha, se han llevado a cabo muchos estudios en el país, especialmente, en las áreas más afectadas por las diferentes formas de leishmaniasis, ubicadas en la región andina y subandina (6-11).

Aunque en la Amazonia y la Orinoquia los estudios han sido escasos, en relación con especies del

\footnotetext{
Correspondencia:

Olga Lucía Cabrera, Grupo de Entomología, Subdirección de Investigación, Instituto Nacional de Salud, Avenida Calle 26 № 51-20, Bogotá, D.C., Colombia.

Teléfono: (0571) 2200923

ocabrera@ins.gov.co, olgalucabrera@yahoo.com

Recibido: 15/05/08; aceptado:24/10/08
}

género Lutzomyia se han reportado 88 (64\%) de las 140 especies registradas en Colombia $(1,12$ 24, Flórez L, Ferro C. Especies de Lutzomyia (Diptera: Psychodidae) encontradas en el municipio de Inírida, departamento de Guainía, Colombia. Memorias, XIII Congreso Colombiano de Parasitología y Medicina Tropical, Ibagué, 1 al 4 de noviembre de 2007), algunas con distribución limitada a estas áreas. El área correspondiente al departamento del Guaviare constituye una zona de transición entre la Amazonia y la Orinoquia (25). Para el departamento del Guaviare, sólo se reporta la presencia de tres especies $L$. choti (14), L. yuilli (16) y L. shawi (15), de las cuales, $L$. shawi corresponde a un error de identificación con L. richardwardi (C. Ferro, comunicación personal).

En la región amazónica la leishmaniasis se presenta de manera esporádica y en su mayoría en ambientes rurales y selváticos, aunque se han reportado algunos casos en áreas periurbanas de Leticia (Ferro C, Pardo R, Díaz A, Pérez L, Munstermann LE. Composición de la fauna de flebotomíneos, género Lutzomyia de la periferia de la ciudad de Leticia, Amazonas Colombia. Memorias, Congreso Internacional Investigación y Salud, Bogotá, 9 al 11 de octubre de 1997). En el departamento de Guaviare, la totalidad de los casos proceden del área rural. Entre 2003 y 2007, se reportaron 1.286 casos de leishmaniasis cutánea en la población civil, de acuerdo con los registros epidemiológicos del Sistema de Vigilancia en Salud Pública (SIVIGILA, consultado el 04 de abril de 2008), con un promedio anual de 251,8 casos y el grupo de edad más afectado (>80\%) corresponde a personas del sexo masculino entre los 15 y 44 años.

Ante la aparición constante de casos de leishmaniasis en la población residente de los municipios del departamento de Guaviare y el poco conocimiento de los flebótomos posibles vectores de parásitos de Leishmania, el Grupo de Entomología del Instituto Nacional de Salud y la Unidad de Entomología de la Secretaría de Salud del Guaviare, iniciaron un estudio entomológico orientado a determinar las especies 
de flebótomos presentes en algunos sectores de los cuatro municipios del departamento y seleccionar entre éstos, por abundancia y antecedentes vectoriales en Latinoamérica, los posibles vectores.

\section{Materiales y métodos \\ Área de estudio}

El departamento del Guaviare está localizado en el extremo norte de la Amazonia colombiana y se extiende desde los $0^{\circ} 32^{\prime}$ hasta los $3^{\circ} 09^{\prime}$ de latitud norte y desde los $69^{\circ} 47^{\prime}$ hasta los $73^{\circ} 47^{\prime}$ longitud occidental. Su extensión es de 42.327 $\mathrm{km}^{2}$ (26), con cuatro municipios: San José del Guaviare, El Retorno, Calamar y Miraflores.

Elclima de la región, deacuerdoconlaclasificación Koppen reportada por el Instituto Geográfico Agustín Codazzi (27), se clasifica como tropical lluvioso de selva isotermal, sin estacionalidad muy acentuada de la precipitación durante todo el año, aunque existe un periodo de menor precipitación durante los meses de diciembre, enero y febrero, y uno de mayor precipitación en los meses de mayo, junio y julio.

En este estudio la captura de flebótomos se realizó en agosto de 2005, abril, mayo y junio de 2006, y agosto y noviembre de 2007, en siete veredas, distribuidas en los municipios del departamento como se muestra en la figura 1: municipio de San José del Guaviare, veredas Capricho, Chuapal, Puerto Cachicamo y Barrancón, ubicadas a los $2^{\circ} 21^{\prime}$ de latitud norte (LN) y $72^{\circ} 49^{\prime}$ longitud occidental (LO), $2^{\circ} 18^{\prime}$ de LN y a los $73^{\circ} 04^{\prime}$ LO, $2^{\circ} 18^{\prime}$ LN y $73^{\circ} 20^{\prime}$ LO y a los $2^{\circ} 36^{\prime}$ de $\mathrm{LN}$ y $72^{\circ} 34^{\prime}$ LO, respectivamente; municipio de El Retorno, veredas Caño Barroso ubicada a los $02^{\circ} 06^{\prime}$ de $L N$ y $72^{\circ} 33^{\prime}$ de LO, Caño Azul $2^{\circ} 05^{\prime}$ LN y $72^{\circ} 32^{\prime}$ LO y Villa Linda LN $2^{\circ} 06^{\prime}$ y LO $72^{\circ} 32^{\prime}$; municipio de Calamar, vereda Candamo a los $1^{\circ} 57^{\prime}$ LN y $72^{\circ} 39^{\prime} \mathrm{LO}$; municipio de Miraflores en el área periurbana $1^{\circ} 20^{\prime} \mathrm{LN}$ y $71^{\circ} 56^{\prime} \mathrm{LO}$. Todos los sitios están por debajo de los $300 \mathrm{msnm}$ y se encuentran a unas tres horas, aproximadamente, de San José del Guaviare por vía terrestre, con excepción del municipio de Miraflores a donde se llega por vía aérea.

\section{Muestreos con trampa CDC}

En el municipio de San José del Guaviare, vereda Chuapal, en un bosque secundario aledaño al caserío sobre el margen izquierdo y derecho de Caño Flauta, se realizaron 56 muestreos con 14 trampas instaladas en un tramo a una distancia entre ellas de $15 \mathrm{~m}$ y a una altura entre los $1,5 \mathrm{y}$ $9 \mathrm{~m}$ durante cuatro noches consecutivas, de las 18:00 a las 06:00 horas. En los otros municipios, en total, se realizaron 13 muestreos en áreas de bosque secundario.

También se realizaron capturas de manera simultánea en el intradomicilio y el peridomicilio en San José del Guaviare, vereda Chuapal (10 viviendas), municipio de Miraflores (6 viviendas) y municipio de El Retorno (6 viviendas). En el municipio de Calamar se instalaron las trampas únicamente en el peridomicilio de tres viviendas. Estos muestreos fueron realizados por dos noches consecutivas.

\section{Capturas con trampa Shannon}

Los muestreos se realizaron a una distancia aproximada de $800 \mathrm{~m}$ de las viviendas en las veredas Chuapal y Villalinda, entre las 19:00 y las 21:30, en el bosque que registró el mayor número de flebótomos capturados con las trampas CDC.

\section{Búsqueda en reposo}

En la vereda Chuapal, en los sitios en donde previamente las trampas CDC detectaron la presencia de flebótomos, se hizo la búsqueda en troncos, grietas y raíces de árboles, entre las 06:00 y las 07:00 durante cuatro días consecutivos, con la ayuda de una linterna para visualizarlos y un aspirador para capturarlos.

\section{Actividad nocturna de picadura}

Se determinó el comportamiento de picadura de los flebótomos en el bosque, en dos sitios separados por una distancia aproximada de 100 $\mathrm{m}$ (en los que previamente se había detectado abundancia de flebótomos con las trampas CDC), de las 18:00 a las 06:00, dos grupos, cada uno constituido por cuatro personas, en dos turnos (18:00 a 01:00 h y 01:00 a 06:00 h) por cuatro noches consecutivas, durante los primeros 50 


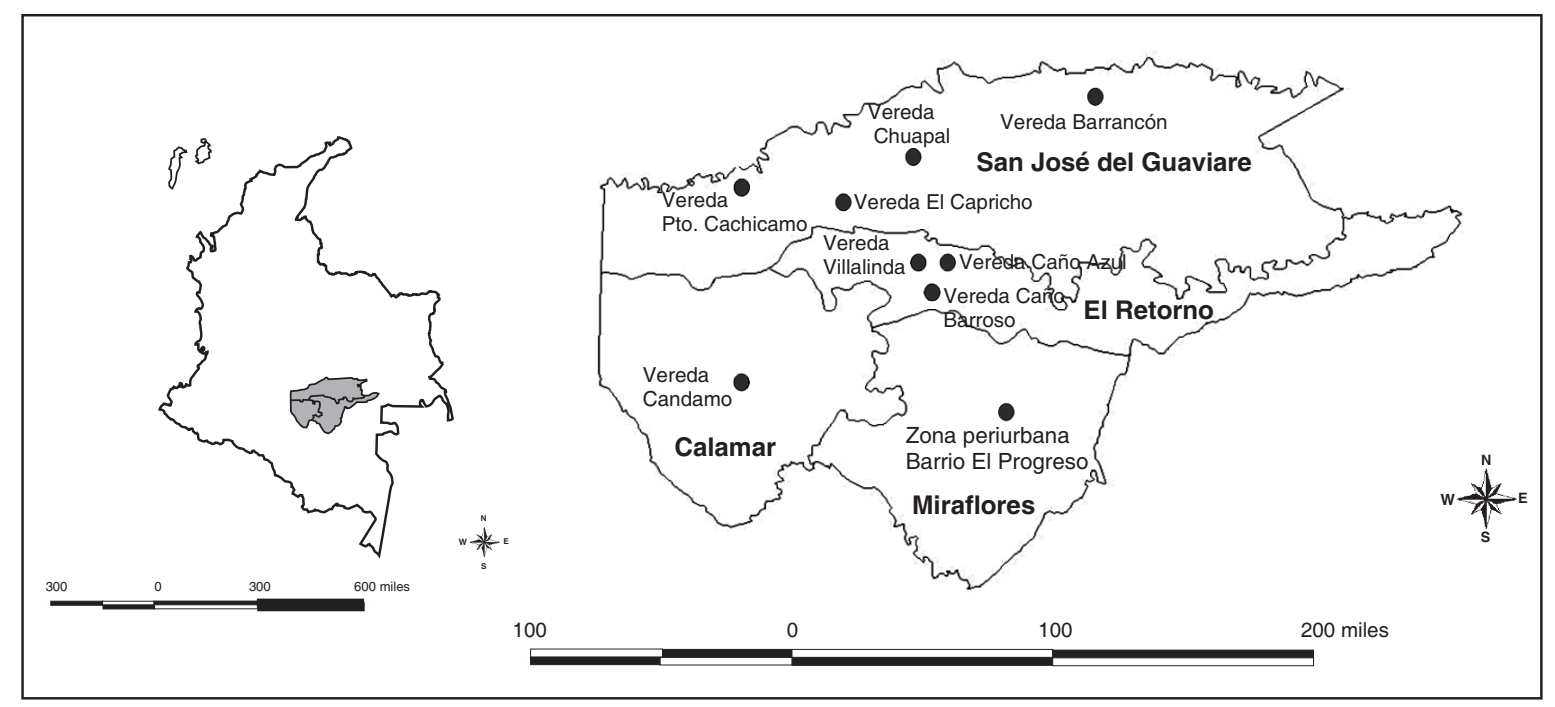

Figura 1. Ubicación de los sitios de muestreo en el departamento del Guaviare.

minutos de cada hora. Los muestreos fueron realizados por personal entrenado quienes usaron prendas que protegían su cuerpo.

\section{Conocimiento sobre la leishmaniasis y sus vectores en los sitios de muestreo}

Se aplicó una encuesta a la comunidad de la vereda Chuapal, para determinar el grado de conocimiento por parte de la población sobre la leishmaniasis y sus vectores.

\section{Determinación de las especies}

Para la determinación taxonómica, los insectos fueron aclarados con $\mathrm{KOH}$ al $10 \%$ y fenol líquido. Se utilizaron las claves de Young (28), Young y Duncan (1), descripciones originales (29-32) y la comparación con ejemplares de la colección de referencia del Grupo de Entomología, Instituto Nacional de Salud. En montaje permanente en bálsamo de Canadá se incluyeron 100 especímenes para fortalecer la colección de referencia del Instituto Nacional de Salud y dar inicio a la colección de referencia de flebótomos de la Unidad de Entomología del Guaviare, con 20 láminas.

\section{Consideraciones éticas}

Las capturas de los flebótomos con atrayente humano fueron realizadas por personas entrenadas que firmaron un consentimiento con conocimiento previo de los objetivos y posibles riesgos de la actividad. De la misma forma, al jefe del hogar donde se instalaron las trampas CDC se le informó el propósito del estudio y dio su consentimiento. Ambos procedimientos fueron aprobados por el Comité de Ética del Instituto Nacional de Salud.

\section{Resultados}

En los 610 ejemplares del género Lutzomyia recolectados se identificaron 37 especies, de las cuales, cuatro se constituyen en nuevos registros para el país (figura 2) y 31 son notificadas por primera vez para la zona de transición entre la Amazonia y la Orinoquia en el departamento del Guaviare (cuadro 1).

Estas especies están distribuidas en 11 subgéneros $y$ tres grupos. Los subgéneros Psychodopygus, Nyssomyia y Sciopemyia, representados por 17 especies (siete, seis y cuatro, respectivamente), agruparon el mayor número de especímenes (504/610; 82,6\%). Las especies más abundantes fueron $L$. hirsuta $24,3 \%(148 / 610)$, L. yuilli $15,2 \%(93 / 610)$ y $L$. davisi $10,3 \%$ (63/610), seguidas por $L$. fartigi, $L$. carrerai (33), $L$. antunesi, $L$. flaviscutellata y $L$. olmeca bicolor, respectivamente (cuadro 1). 
Cuadro 1. Especies de Lutzomyia por municipio y localidad en el departamento del Guaviare.

\begin{tabular}{|c|c|c|c|c|c|c|c|c|c|c|c|c|c|c|c|c|c|c|c|c|}
\hline & \multicolumn{9}{|c|}{ San José del Guaviare } & \multicolumn{6}{|c|}{ EI Retorno } & \multirow{2}{*}{\multicolumn{2}{|c|}{$\frac{\text { Calamar }}{\text { Candamo }}$}} & \multirow{2}{*}{\multicolumn{2}{|c|}{$\frac{\text { Miraflores }}{\text { Progreso }}$}} & \multirow{3}{*}{ Total } \\
\hline & \multicolumn{2}{|c|}{ Chuapal } & \multicolumn{2}{|c|}{ Capricho } & \multicolumn{2}{|c|}{$\begin{array}{c}\text { Puerto } \\
\text { Cachicamo }\end{array}$} & \multicolumn{3}{|c|}{ Barrancón } & \multicolumn{2}{|c|}{ Caño Azul } & \multicolumn{2}{|c|}{ Caño Barroso } & \multicolumn{2}{|c|}{ Villa Linda } & & & & & \\
\hline & 우 & 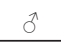 & 운 & 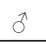 & q & 0 & q & 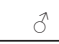 & & 우 & $\delta$ & q & 8 & q & $\hat{0}$ & 운 & 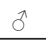 & 운 & $\delta$ & \\
\hline Aragaoi & & & & & & & & & & & & & & & & & & & & \\
\hline L. aragaoi* & - & - & - & 1 & - & - & - & - & - & - & - & - & - & - & - & - & - & - & - & 1 \\
\hline L. barrettoi majuscula* & - & 2 & - & 1 & - & - & - & - & - & - & - & - & - & - & - & - & - & - & - & 3 \\
\hline L. barretoi barretoi* & - & 2 & - & - & - & - & - & - & - & - & - & - & - & - & - & - & - & - & - & 2 \\
\hline $\begin{array}{l}\text { L. carpenteri* } \\
\text { Evandromyia }\end{array}$ & - & - & - & - & - & - & - & - & - & 2 & - & - & - & - & - & 1 & - & - & - & 3 \\
\hline $\begin{array}{l}\text { L. begonae }{ }^{* *} \\
\text { Lutzomyia }\end{array}$ & 1 & - & - & - & - & - & - & - & - & - & - & - & - & - & - & 11 & - & - & - & 12 \\
\hline L. evangelistai* & 2 & - & - & - & - & - & - & - & - & - & - & - & - & - & - & - & - & - & - & 2 \\
\hline $\begin{array}{l}\text { L. gomezi* } \\
\text { Micropygomyia }\end{array}$ & - & - & - & - & - & - & - & - & - & - & - & - & - & - & 1 & - & - & - & - & 1 \\
\hline $\begin{array}{l}\text { L. micropyga } \\
\text { Migonei }\end{array}$ & 1 & - & - & - & - & - & - & - & - & - & - & - & - & - & - & - & - & - & - & 1 \\
\hline L. sericea** & - & - & - & - & - & - & - & - & - & - & - & - & - & 1 & - & - & - & - & - & 1 \\
\hline L. walkeri* & - & - & - & - & - & - & - & - & 1 & - & - & - & - & - & - & - & - & 3 & 1 & 5 \\
\hline $\begin{array}{l}\text { L. sp. de Badue/* } \\
\text { Nyssomyia }\end{array}$ & - & - & - & - & - & - & - & - & - & - & - & - & - & - & - & 1 & - & - & - & 1 \\
\hline L. antunesi* & 3 & 3 & - & - & - & - & - & - & - & - & - & - & - & - & - & 5 & - & 2 & 4 & 17 \\
\hline L. flaviscutellata* & 7 & 2 & 3 & - & - & - & . & - & - & 1 & - & - & - & - & - & 1 & - & 1 & - & 15 \\
\hline L. olmeca bicolor & 13 & 2 & - & - & - & - & . & - & - & - & - & - & - & - & - & - & - & - & - & 15 \\
\hline L. pajoti* & - & - & - & - & - & - & - & - & - & - & - & - & - & 4 & 2 & - & - & - & - & 6 \\
\hline L. richardwardi* & 1 & - & - & - & - & - & - & - & - & - & - & - & - & 1 & 1 & - & - & - & - & 3 \\
\hline $\begin{array}{l}\text { L. yuilli } \\
\text { Pressatia }\end{array}$ & 86 & 2 & - & - & - & - & - & - & - & - & - & - & - & 1 & - & 4 & - & - & - & 93 \\
\hline $\begin{array}{l}\text { L. dysponeta* } \\
\text { Psathyromyia }\end{array}$ & - & - & - & - & - & - & - & - & - & - & 2 & - & 1 & - & 1 & - & - & - & - & 4 \\
\hline L. campbelli** & - & - & - & - & - & - & - & - & - & 1 & - & - & - & - & - & - & - & - & - & 1 \\
\hline L. scaffi & 1 & - & - & - & - & - & - & - & - & - & - & - & - & - & - & - & - & - & - & 1 \\
\hline L. shannoni* & - & - & - & - & - & - & - & - & - & 2 & - & - & - & - & - & - & - & - & - & 2 \\
\hline $\begin{array}{l}\text { L. sp. }{ }^{*} \\
\text { Psychodopygus }\end{array}$ & 1 & - & - & - & - & - & - & - & - & - & - & - & - & - & - & - & - & - & - & 1 \\
\hline L. carrerai ${ }^{\star}$ & 13 & 4 & - & - & - & - & - & - & - & 2 & 6 & - & - & - & - & - & - & - & - & 25 \\
\hline L. davisi* & 20 & 12 & - & - & - & - & - & - & - & 12 & 5 & - & - & 5 & 1 & 8 & - & - & - & 63 \\
\hline L. fairtigi ${ }^{*}$ & 3 & 2 & - & 1 & - & - & - & - & - & 14 & 6 & - & - & 12 & 9 & 4 & - & - & - & 51 \\
\hline L. hirsuta * & 62 & 61 & - & 1 & - & 2 & - & - & - & 5 & 3 & - & - & 7 & 5 & 2 & - & - & - & 148 \\
\hline L. panamensis ${ }^{\star}$ & 3 & - & - & - & - & - & - & - & - & - & - & - & - & - & - & - & - & - & - & 3 \\
\hline L. sp. de Tres Esquinas* & - & - & - & - & - & - & - & - & - & 1 & - & - & - & - & - & - & - & - & - & 1 \\
\hline L. geniculata* & 8 & 3 & - & - & - & - & - & - & - & - & - & - & - & - & - & - & - & 7 & 4 & 22 \\
\hline $\begin{array}{l}\text { L. geniculata guyanensis } \\
\text { Saulensis }\end{array}$ & 2 & - & - & - & - & - & - & - & - & - & - & - & - & - & - & - & - & - & - & 2 \\
\hline $\begin{array}{l}\text { L. saulensis* } \\
\text { Sciopemyia }\end{array}$ & - & 1 & - & - & - & - & - & - & - & - & - & - & - & - & - & 2 & - & - & - & 3 \\
\hline L. preclara* & - & 3 & - & - & - & - & - & - & - & - & 2 & - & - & - & - & - & - & 2 & - & 7 \\
\hline L. vattierae* & 5 & 1 & - & - & 1 & - & - & - & - & 2 & - & - & - & 1 & - & 1 & 1 & - & - & 12 \\
\hline L. sordelli* & & 1 & - & - & - & - & - & - & - & 2 & - & - & - & 1 & - & 5 & - & - & - & 9 \\
\hline $\begin{array}{l}\text { L. nematoducta** } \\
\text { Trichophoromyia }\end{array}$ & 10 & - & 1 & - & - & - & - & - & - & 1 & - & - & - & - & - & - & - & $\begin{array}{l}- \\
-\end{array}$ & - & 12 \\
\hline L. cellulana* & - & 23 & - & - & - & 2 & - & - & - & - & 7 & - & - & - & 1 & - & - & - & - & 33 \\
\hline $\begin{array}{l}\text { L. sp.* } \\
\text { Trichopygomyia }\end{array}$ & 11 & - & - & - & - & - & - & - & - & 3 & - & - & - & - & - & 4 & - & 2 & - & 20 \\
\hline $\begin{array}{l}\text { L. witoto* } \\
\text { Viannamyia }\end{array}$ & - & - & - & - & - & - & - & - & - & 1 & 1 & - & - & 2 & - & - & - & - & - & 4 \\
\hline L. caprina* & 2 & - & - & - & - & - & - & - & - & - & - & - & - & - & - & - & - & - & - & 2 \\
\hline L. tuberculata* & 1 & - & - & - & - & - & - & - & - & - & - & - & - & - & - & - & - & - & - & 1 \\
\hline Brumptomyia* & 2 & - & - & - & - & - & - & - & - & - & - & - & - & - & - & - & - & - & - & 2 \\
\hline Total & 259 & 123 & 4 & 4 & 1 & 4 & 0 & 0 & 1 & 49 & 32 & 0 & 1 & 35 & 21 & 49 & 1 & 17 & 9 & 610 \\
\hline
\end{tabular}

* Nuevos registros para el departamento

** Nuevos registros para el país 

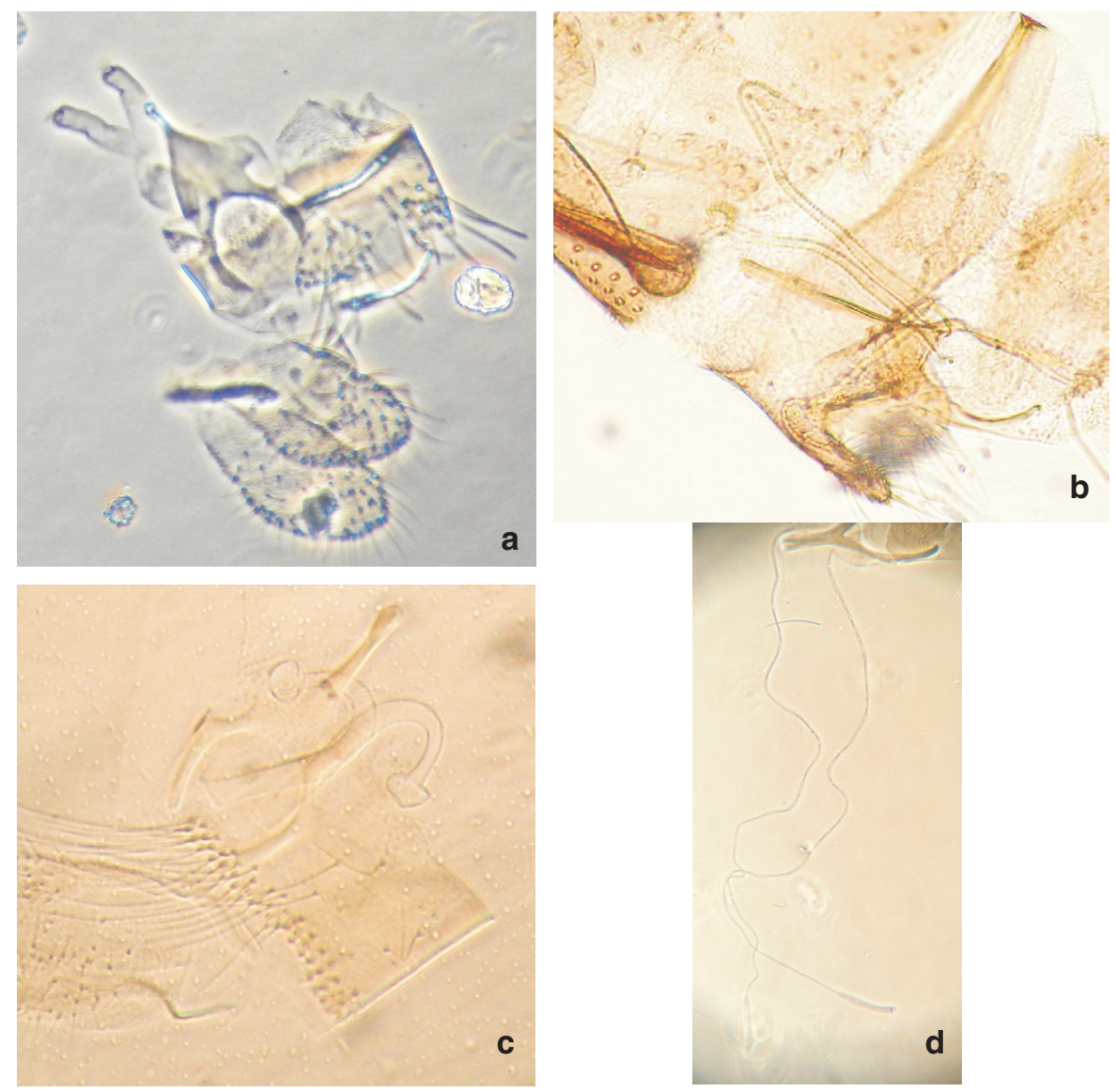

Figura 2. Espermatecas de especies de Lutzomyia, nuevos registros para el país: a. L. begonae (40X), b. L. campbelli (10X), c. L. sericea (40X), d. L. nematoducta (4X).

En las trampas CDC instaladas en el interior del bosque (56 muestreos), a una altura promedio de $7,3 \mathrm{~m}$, se recolectó aproximadamente el $76 \%$ del total de los flebótomos identificados en este estudio. El hallazgo de un macho de L. witoto y una hembra del subgénero Trichopygomyia en la misma localidad, permitió la identificación del ejemplar hembra como L. witoto.

Delos 14 intradomicilios muestreados únicamente cuatro fueron positivos para Lutzomyia, donde en total se encontraron tres machos de L. cellulana, tres hembras del subgénero Trichophoromyia y una hembra de $L$. vattierae. El peridomicilio en las veredas Chuapal y Caño Azul fue positivo con la captura de dos hembras de L. hirsuta, mientras que en el municipio de Miraflores se recolectaron 26 flebótomos distribuidos en seis especies: L. walkeri, L. antunesi, L. flaviscutellata, L. geniculata, L. preclara y $L$. subgénero Trichophoromyia.

En sitio de reposo, tronco de árbol, se encontraron dos especies del subgénero Viannamyia: $L$. furcata y L. tuberculata.

Con respecto al comportamiento de picadura realizado dentro del bosque, el total de hembras del género Lutzomyia capturadas con atrayente humano fue de 43 , distribuidas en las siguientes especies: L. carrerai (24ㅇ), L. yuilli (8ㅇ), L. davisi (7ㅇ), L. hirsuta (3) y L. scaffi (1+). L. carrerai fue activa en un amplio rango de la noche y la madrugada, desde las 18:00 a las 04:00, con 
una máxima actividad entre las 21:00 y las 22:00 $\mathrm{h}$, con un promedio de 1,4 hembras/persona por hora. L. yuilli se acercó a picar después de las 24:00 y fue más frecuente entre las 01:00 y las 02:00, con un promedio de 0,4 hembras/persona por hora. L. davisi fue activa especialmente en la madrugada. En las otras dos especies no se pudo observar un patrón de actividad porque el número de hembras capturadas fue muy reducido.

La encuesta se realizó en las 13 viviendas de la vereda Chuapal, en donde vivían 100 personas, aproximadamente, con un promedio de permanencia en el sitio de 4,5 años. Se pudo establecer que todos los jefes de hogar entrevistados conocían la enfermedad y la designaban con el nombre de leishmaniasis. Sólo dos jefes de hogar de los 13 entrevistados reconocían los flebótomos y los llamaban capotillo y mantablanca; es importante aclarar que estas dos personas provenían del área rural de los departamentos de Tolima y Huila, donde también es endémica la leishmaniasis. Finalmente, ningún encuestado asoció a los flebótomos con la transmisión de la enfermedad; tampoco se registró molestia causada por la picadura de estos insectos.

\section{Discusión}

Del total de especies identificadas en este estudio, se registran por primera vez para el país: L. begonae, L. campbelli, L. sericea y $L$. nematoducta. L. begonae estaba reportada para Venezuela, L. campbelli para Bolivia, Brasil, Guyana Francesa y Venezuela, L. sericea para Brasil, Ecuador, Guyana Francesa y Venezuela, y L. nematoducta para Brasil $(1,34)$. L. yuilli y $L$. richardwardi se habían encontrado en la zona específica de este estudio (Guaviare). $L$. carpenteri, L. evangelistai, L. pajoti, L. scaffi, $L$. davisi, $L$. witoto y $L$. furcata sólo en la Amazonia y $L$. dysponeta y $L$. vattierae en la Orinoquia. Las especies restantes han sido registradas tanto en la Amazonia como en la Orinoquia (cuadro 2).

Cuadro 2. Especies del género Lutzomyia (Diptera: Psychodidae) registradas en los departamentos de la Orinoquia y la Amazonia colombiana

Grupo Aragaoi Theodor, 1965

L. abunaensis Martins Falção \& Silva, 1965: Amazonas, Leticia km 17 (16).

L. aragaoi (Costa Lima, 1932): Amazonas; Caquetá (15); Casanare, Tauramena (a); Guainía, Inírida (b); Guaviare*, San José del Guaviare Inspección Capricho; Putumayo y Vichada (15).

L. barretoi barretoi (Mangabeira, 1942): Caquetá (15); Guaviare ${ }^{\star \star}$, San José del Guaviare vereda Chuapal; Meta, vereda La Reforma Villavicencio (c).

L. barretoi majuscula Young, 1979: Meta, vereda La Reforma Villavicencio (c), Guaviare*, San José del Guaviare vereda Capricho y Chuapal.

L. carpenteri (Fairchild \& Hertig. 1953): Guainía, Inírida (b); Guaviare**, Calamar vereda Candamo, El Retorno vereda Caño Azul.

L. runoides (Fairchild \& Hertig. 1953): Amazonas, Leticia km 17 (28,15); Caquetá, Araracuara, Puerto Abejas (19).

Grupo Dreisbachi Lewis, 1977

L. dreisbachi (Causey \& Damasceno, 1945): Amazonas, Leticia km 17 y Caquetá, Araracuara (13).

Subgénero Evandromyia Mangabeira, 1941

L. begonae Ortiz y Torres 1975: Guaviare**, Calamar vereda Candamo, San José del Guaviare vereda Chuapal.

L. infraspinosa (Mangabeira, 1941): Amazonas, Leticia km $17(15,35)$.

L. monstruosa (Floch \& Abonnenc, 1941): Amazonas, Leticia km 17 (15) y Caquetá, Araracuara (13).

Subgénero Helcocyrtomyia Barretto, 1962

L. tortura Young \& Rogers, 1984: Amazonas, Parque Nacional Amaca-Yacú (14); Putumayo, Orito, Puerto Asís, Puerto Caicedo, Puerto Gúzman, Puerto Leguízamo, San Miguel, Valle del Guamuez, Villa Garzón (17).

Subgénero Lutzomyia França, 1924

L. araracuarensis Morales \& Minter 1981: Amazonas (15) y Caquetá, Araracuara (13).

L. evangelistai Martins \& Fraiha, 1971: Amazonas (15); Guaviare**, San José del Guaviare vereda Chuapal; Putumayo, Mocoa, Puerto Asís, Puerto Caicedo, Villa Garzón (17). 
L. falcata Young, Morales \& Ferro, 1994: Amazonas (15).

L. gomezi (Nitzulescu, 1931): Amazonas, Leticia km 17 (28); Arauca, Tame (d); Caquetá, Araracuara (13), Tres Esquinas (28), Guaviare**, El Retorno vereda Villa Linda; Meta, Villavicencio veredas San Antonio, Quenane y La Reforma (c,12,36); Vichada, Cumariana (12).

L. lichyi Floch y Abonnenc 1950: Meta, Macarena y Villavicencio vereda San Antonio $(36,37)$.

L. marinkelli Young, 1979: Amazonas (15), Caquetá, Araracuara (13), Tres Esquinas (28).

L. sherlocki Martins, Silva \& Falção 1971: Amazonas, Leticia km 17 (28), ribera río Pupuña, afluente del río Putumayo (18).

Grupo Migonei Theodor, 1965

L. dubitans (Sherlock, 1962): Arauca, Tame (d)

L. migonei (França, 1920): Casanare, Tauramena (a).

L. sericea Floch y Abonnenc 1944: Guaviare ${ }^{\star \star \star}$, El Retorno vereda Villa Linda.

L. sp. de Baduel (Floch \& Abonnenc 1945): Caquetá, Solano, Tres Esquinas (28); Guaviare**, Calamar vereda Candamo; Vichada, Cumariana (12).

L. walkeri (Newstead, 1914): Amazonas (15), Labrea (28), 1 km de Leticia (e); Caquetá, Tres Esquinas, Solano vereda La (28); Casanare, Tauramena (a); Guaviare ${ }^{\star *}$, Miraflores El Progreso, San José del Guaviare vereda Barrancón; Meta, San Martín (36), Villavicencio vereda La Reforma (c); Putumayo, Puerto Asís, Puerto Leguízamo, Villagarzón (17) y Vaupés (16).

\section{Subgénero Micropygomyia Barretto, 1962}

L. micropyga (Mangabeira, 1942): Amazonas (15); Casanare, Tauramena (a), Guaviare**, San José del Guaviare vereda Chuapal; Meta; Putumayo, Puerto Asís (17).

L. venezuelensis (Floch \& Abonnenc, 1948): Meta, Acacías, vereda Quebrada Cola de Pato (12); Vaupés (15).

\section{Subgénero Nyssomyia Barretto, 1962}

L. antunesi (Coutinho, 1939): Amazonas, Leticia km 1 (e); Arauca, Tame (d); Caquetá, Araracuara, Florencia vereda Milán, Solano (12,13); Casanare, Tauramena (a); Guainía, Inírida (b,38); Guaviare**, Calamar vereda Candamo, Miraflores El Progreso, San José del Guaviare vereda Chuapal; Meta, Puerto Gaitán, veredas San Pedro de Arimena y Carimagua, (36), Puerto Concordia vereda La Cascada (Datos, Grupo de Entomología, INS, 2007), San Martín (36), Villavicencio veredas La Reforma (c) y San Antonio (36); Vaupés (16) y Vichada, Cumariana (12).

L. flaviscutellata (Mangabeira, 1942): Amazonas (15); Casanare, Tauramena (a), Guaviare ${ }^{\star *}$, Calamar vereda Candamo, El Retorno vereda Caño Azul, Miraflores barrio El Progreso, San José del Guaviare Inspección Capricho y vereda Chuapal.

L. olmeca bicolor Fairchild \& Theodor, 1971: Amazonas (15); Caquetá; Casanare, Tauramena (a); Guainía, Inírida (b,38);

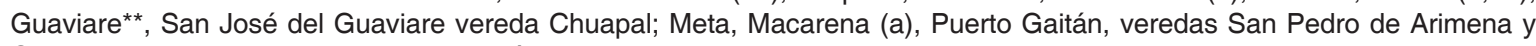
Carimagua, (36); Putumayo, Puerto Leguízamo (17).

L. pajoti Abonnenc, Leger y Fauran, 1979: Guaviare ${ }^{\star \star}$, El Retorno vereda Villa Linda; Putumayo, Orito, Puerto Asís, Puerto Caicedo, Puerto Guzmán, Puerto Leguízamo, Villagarzón (17).

L. reducta Feliciangeli, Ramírez-Pérez \& Ramírez, 1988: Amazonas, Leticia km 8 (e), Ribera río Pupuña, afluente del río Putumayo (18).

L. richardwardi Ready \& Fraiha, 1981: Amazonas (15), Ribera río Pupuña, afluente del río Putumayo (18), Guaviare*, EI Retorno vereda Villa Linda y San José del Guaviare vereda Chuapal; Meta, Río Iteviare (14); Putumayo, Pto. Caicedo, Pto. Leguízamo, Villagarzón (17), Vaupés (15).

L. umbratilis Ward \& Fraiha, 1977: Amazonas (15), Leticia km 17 (28), km 1 de Leticia (e), ribera río Pupuña, afluente del río Putumayo (18); Caquetá, Araracuara (13) y Putumayo, Puerto Caicedo, Puerto Guzmán, Puerto Leguízamo, Villagarzón (17).

L. yuilli Young \& Porter, 1972: Amazonas, Leticia km 1 (e); Caquetá, Araracuara, Puerto Abejas (13,19); Guainía, Inírida (b); Guaviare*, Calamar vereda Candamo, El Retorno vereda Villa Linda, San José del Guaviare vereda Chuapal; Meta, Macarena (37), Mapiripán, Rincón Indio Grupo de Entomología, San Martín (12,36); Putumayo, Mocoa, Orito, Puerto Asís, Puerto Caicedo, Puerto Guzmán, San Miguel, Valle del Guamuez, Villagarzón (17).

\section{Grupo Oswaldoi Theodor, 1965}

L. rorotaensis (Floch \& Abonnenc, 1944): Meta, Puerto Concordia vereda La Cascada (Grupo de Entomología 2007).

L. sp. 2 de Araracuara Morales y Minter, 1981: Caquetá, Araracuara (13).

L. trinidadensis (Newstead, 1922): Meta, Villavicencio, Quenane (36).

Grupo Pilosa Theodor, 1965

L. chassigneti (Floch \& Abonnenc, 1944): Amazonas, Leticia km 1 (16); Caquetá, Araracuara (13).

L. pilosa (Damasceno \& Causey, 1944): Amazonas (15); Caquetá, Florencia vereda Milán (12); Putumayo, Villagarzón (17).

Subgénero Pintomyia Costa Lima, 1932

L. christenseni Young \& Duncan, 1994: Amazonas; Caquetá (15); Casanare, Tauramena (a); Meta (15).

L. damascenoi (Mangabeira, 1941): Caquetá, Puerto Abejas (19), Meta (15).

Subgénero Pressatia Mangabeira, 1924

L. choti (Floch \& Abonnenc, 1941): Meta, Macarena (a); Guaviare*, San José del Guaviare vereda Caño Ovejas (14). 
L. dysponeta (Fairchild \& Hertig, 1953): Guaviare*, El Retorno veredas Caño Azul y Villa Linda y Meta Macarena (a). L. triacantha (Mangabeira, 1942): Meta, Mapiripán y Puerto Lleras (28).

Subgénero Psathyromyia Barretto, 1962

L. abonnenci (Floch \& Chassingnet, 1947): Caquetá, Solano vereda La Nevera (12); Meta, Macarena (a).

L. cuzquena Martins, Llanos \& Silva, 1975: Amazonas (15), km 8 de Leticia (14).

L. dendrophyla (Mangabeira, 1942): Amazonas, ribera río Pupuña, afluente del río Putumayo (18); Caquetá, Araracuara, Florencia en Belén y Milán, Solano vereda La Nevera (12,13); Meta, Acacías, veredas Guarupaya y Quebrada Cola de Pato, Fuente de Oro inspección Puerto Limón, Guamal, y en San Martín vereda Sabana de San Martín (12); Putumayo, Puerto Caicedo, Puerto Leguízamo, Valle del Guamuez, Villagarzón (17) y Vaupés (15).

L. Iutziana (Costa Lima, 1932): Amazonas, Leticia km 9 (14); Meta (39).

L. punctigeniculata (Floch \& Abonnenc, 1944): Casanare, Tauramena (a); Caquetá, Solano vereda La Nevera (12); Putumayo, Puerto Caicedo (17).

L. scaffi Damasceno \& Arouk, 1956: Amazonas (15) y Caquetá, Araracuara (13).

L. shannoni (Dyar, 1929): Amazonas, ribera río Pupuña, afluente del río Putumayo (18); Caquetá Florencia en Belén y Milán; Guaviare $^{\star \star}$, El Retorno veredas Caño Azul y Villa Linda y Meta, Macarena (a).

L. campbelli Damasceno, Causey y Arouck 1945: Guaviare***, El Retorno vereda Caño Azul.

\section{Subgénero Psychodopygus Mangabeira, 1941}

L. amazonensis (Root, 1934): Amazonas (15); Caquetá, Araracuara (13); Meta, Macarena (15,37); Putumayo, Puerto Asís, Puerto Caicedo, Puerto Leguízamo, San Miguel (17).

L. ayrozai (Barretto \& Coutinho, 1940): Amazonas (15) Guainía, Inírida (b); Meta, Macarena (37).

L. bernalei (Osorno, Morales \& de Osorno, 1967): Caquetá, Araracuara (13); Guainía Inírida (b).

L. bispinosa (Fairchild \& Hertig, 1951): Amazonas (15); Caquetá, Araracuara (13); Putumayo, Puerto Caicedo (17) y Vaupés (15).

L. carrerai (Barretto, 1946): Amazonas, ribera río Pupuña, afluente del río Putumayo (18); Caquetá, Puerto Abejas (19); Guaviare $^{\star *}$, El Retorno vereda Caño Azul y San José del Guaviare vereda Chuapal; Meta, Macarena (39), Restrepo (12) y Putumayo, Puerto Asís, Puerto Caicedo, Puerto Leguízamo, Valle del Guamuez (17).

L. chagasi (Costa Lima, 1941): Amazonas, ribera río Pupuña, afluente del río Putumayo (18,12); Caquetá, Araracuara, Puerto Abeja (a,13); Meta, Villavicencio vereda La Reforma (c); Putumayo, Villagarzón (17); Vaupés (15).

L. claustrei Abonnenc, Leger \& Fauran, 1979: Guainía, Inírida (b); Meta, río Iteviare (14); Putumayo, Puerto Asís, Puerto Caicedo, Puerto Guzmán (17).

L. davisi (Root, 1934): Amazonas, Leticia km 18 (28), río Pupuña (18); Caquetá, Araracuara (13), Florencia vereda Milán (12), Tres Esquinas (28); Guainía, Inírida (b,38); Guaviare**, Calamar vereda Candamo, El Retorno veredas Caño azul y Villa Linda, San José del Guaviare vereda Chuapal; Meta, Macarena (37); Putumayo, Orito, Puerto Asís, Puerto Caicedo, Puerto Leguízamo, San Miguel, Valle del Guamuez, Villagarzón (17).

L. fairtigi Martins, 1970 (40): Caquetá, Tres Esquinas (28); Casanare, Tauramena (a); Guaviare**, El Retorno vereda Caño Azul, vereda Villa Linda, Calamar vereda Candamo, San José del Guaviare Inspección Capricho, vereda Chuapal); Meta, Villavicencio (12), Vista Hermosa, Las TIgres (36); Vaupés (15).

L. geniculata (Mangabeira, 1941): Amazonas, ribera río Pupuña (18); Caquetá, Tres Esquinas (28), Araracuara (18), Solano, vereda La Nevera, El Palmar (12), Guaviare**, Miraflores El Progreso y San José del Guaviare vereda Chuapal; Meta (15); Puerto Concordia, vereda. La Cascada (Datos Grupo de Entomología, INS, 2007), Putumayo, Orito, Puerto Asís, Puerto Caicedo, Puerto Leguízamo, Valle del Guamuez (17).

L. hirsuta (Mangabeira, 1942): Amazonas, ribera río Pupuña, afluente del río Putumayo (18), Caquetá, Guaviare ${ }^{\star \star}$, Calamar vereda Candamo, El Retorno vereda Caño Azul y Villa Linda y San José del Guaviare Inspección Capricho, veredas Chuapal y Puerto Cachicamo; Meta, Macarena (a); Meta, Macarena (37); Putumayo, Orito, Puerto Asís, Puerto Caicedo, Puerto Leguízamo, San Miguel, Valle del Guamuez, Villagarzón (17) y Vaupés (15).

L. nocticola Young, 1973: Amazonas, río Pupuña (18); Casanare, Tauramena (a); Putumayo, Puerto Caicedo (17).

L. panamensis (Shannon, 1926): Caquetá, Tres Esquinas (28); Casanare, Tauramena (a); Guaviare**, San José del Guaviare vereda Chuapal; Meta, Restrepo, Balcones (36), Villavicencio, vereda Aguas Claras (12), vereda La Reforma (c).

L. paraensis (Costa Lima, 1941): Amazonas, ribera río Pupuña, afluente del río Putumayo (18); Caquetá, Araracuara (13) y Putumayo, Puerto Caicedo, Puerto Leguízamo (17).

L. sp. de Tres Esquinas Young 1979: Caquetá, Tres Esquinas (28), Araracuara (13); Guaviare**, El Retorno, Caño Azul; Meta, Puerto Concordia, vereda. La Cascada (datos Grupo de Entomología INS, 2007); Putumayo, Puerto Leguízamo, Villagarzón (17).

\section{Grupo Saulensis Lewis et al., 1977}

L. saulensis (Floch \& Abonnenc, 1944): Amazonas (15); Caquetá, Solano vereda La Nevera, Puerto Abejas (12, 28, 19),

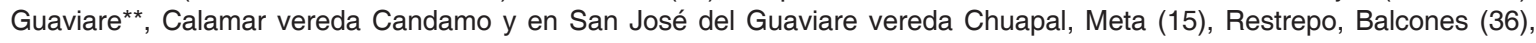
Villavicencio La Reforma (c), Puerto Concordia vereda La Cascada (datos Grupo de Entomología INS 2007), Putumayo (L. saulensis sp.) Puerto Caicedo, Puerto Leguízamo (17). 


\section{Subgénero Sciopemyia Barretto, 1962}

L. preclara Young \& Arias, 1984: Amazonas (15); Guaviare**, El Retorno vereda Caño Azul, Miraflores Progreso, San José del Guaviare, Chuapal; Meta, río Iteviare (14), Mapiripán Rincón Indio (datos Grupo de Entomología INS).

L. sordelli (Shannon \& Del Ponte, 1927): Amazonas, Leticia km 17 (28), Caquetá, Solano vereda La Nevera (12), Araracuara (13); Guaviare**, Calamar, vereda Candamo, El Retorno, vereda Caño Azul, vereda Villa Linda, San José del Guaviare, Chuapal; Meta (15); Putumayo, Puerto Leguízamo (17).

L. vattierae Le Pont y Desjeux, 1992: Guaviare**, Calamar vereda Candamo, El Retorno, veredas Caño Azul, Villa Linda, San José del Guaviare veredas Chuapal y Puerto Cachicamo; Meta, La Macarena, Los Andes (37); Villavicencio, La Reforma (c). L. nematoducta Young \& Arias, 1981: Guaviare***, El Retorno, vereda Caño Azul, San José del Guaviare vereda Chuapal, Inspección Capricho.

\section{Subgénero Trichophoromyia Barretto, 1962}

L. auraensis (Mangabeira, 1942): Caquetá, Solano vereda La Nevera (12); Putumayo, Puerto Asís, Puerto Leguízamo, San Miguel, Valle del Guamuez (17).

L. bettini Feliciangeli, Ramírez Pérez y Ramírez, 1988: Guainía, Inírida (b).

L. cellulana Young, 1979: Caquetá (15); Meta, Macarena (a), Puerto Concordia vereda La Cascada (datos Grupo de Entomología INS 2007); Guaviare ${ }^{\star \star}$, El Retorno vereda Caño Azul y Villa Linda, San José del Guaviare veredas Chuapal y Puerto Cachicamo; Putumayo, Puerto Leguízamo y Valle del Guamuez (17).

L. howardi Young, 1979: Amazonas Leticia km 1 (16), ribera río Pupuña, afluente del río Putumayo (18); Putumayo, Puerto Leguízamo (17).

L. ruii Arias y Young, 1982: Amazonas (16).

L. pabloi Barreto, Burbano y Young, 2002: Putumayo, Puerto Asís veredas Buena Vista y Belén, Villagarzón vereda San Vicente (20).

L. saltuosa Young, 1979: Amazonas (15).

L. ubiquitalis (Mangabeira, 1942): Caquetá, Florencia vereda Milán (12) y Meta (15).

L. sp. $\mathbf{N}^{\circ} 1$ de Araracuara: Caquetá, Araracaura (13).

Subgénero Trichopygomyia Barretto, 1962

L. conviti Ramírez, Pérez, Martins \& Ramírez, 1976: Meta, río Iteviare (14).

L. longispina (Mangabeira, 1942): Caquetá, Araracuara (13); Meta, Macarena (a).

L. martinezi Young \& Morales, 1987: Meta, río Iteviare (14).

L. wagleyi Morales y Minter, 1981: Caquetá, Araracuara (13).

L. witoto Young \& Morales, 1987: Amazonas, Leticia km 8 (14); Guaviare**, El Retorno veredas Caño Azul y Villa Linda; Putumayo, Puerto Caicedo, Puerto Leguízamo, Villagarzón (17).

Grupo Verrucarum Theodor, 1965

L. evansi (Nuñez-Tovar, 1924): Meta, Macarena (a).

L. nevesi (Damasceno \& Arouck, 1956): Amazonas, Parque Nacional Amaca-Yacú (14), río Pupuña (18); Caquetá (15); Meta (15) y Putumayo, Puerto Asís (17).

L. serrana (Damasceno \& Arouck, 1949): Meta, Restrepo (36,37).

Subgénero Viannamyia Mangabeira, 1941

L. furcata (Mangabeira, 1941): Amazonas (16); Guaviare**, San José del Guaviare vereda Chuapal.

L. tuberculata (Mangabeira, 1941): Caquetá, Araracuara (12); Guaviare ${ }^{\star \star}$, San José del Guaviare vereda Chuapal y Meta, Macarena (a).

a. Molina J, Jaramillo M, Villegas C, Guhl F. Actualización de la distribución del género Lutzomyia en Colombia. IX Congreso Colombiano de Parasitología y Medicina Tropical Medellín 1997. p. 152-3.

b. Flórez L, Ferro C. Especies de Lutzomyia (Díptera: Psychodidae) encontradas en el municipio de Inírida, departamento de Guainía, Colombia. Memorias XIII Congreso Colombiano de Parasitología y Medicina Tropical, Ibagué. Biomédica 2007:27(Suppl. 2):222.

c. Vásquez A, González A, Santamaría E, Góngora A, Cabrera OL, Buitrago LE. Detection of Leishmania spp. in canines and species of the genus Lutzomyia in a rural area of Villavicencio, Colombia. XVII American Mosquito Control Association, Orlando, Florida 1 al 5 de abril de 2007.

d. Turriago B, Ferro C, Rodríguez H, Castro F, Cuervo L. Primer registro de Lutzomyia (Diptera: Psychodidae) en el departamento de Arauca. XXXIV Congreso de Entomología Sociedad Colombiana de Entomología Cartagena de Indias, Colombia julio 25, 26 y 27 de 2007. p. 120-1.

e. Ferro C, Pardo R, Díaz A, Pérez L, Munstermann L. Composición de la fauna de flebotomíneos, género Lutzomyia, de la periferia de la ciudad de Leticia, Amazonas, Colombia. Congreso Internacional Investigación y Salud, Bogotá. Biomédica 1997;17:84.

\footnotetext{
${ }^{*}$ Registro para el departamento del Guaviare

** Nuevo registro para el departamento del Guaviare.

*** Nuevo registro para el país

No se incluye a $L$. shawi en la lista, porque se considera un error de identificación que debe ser confirmado.
} 
L. hirsuta y L. yuilli, de los subgéneros Psychodopygus y Nyssomyia, fueron las dos especies más abundantes, lo que coincide con el comportamiento descrito por Arias y Freitas (41) para la Amazonia central brasilera, en el cual las especies de los subgéneros Psychodopygus y Nyssomyia presentaban una distribución restringida a áreas boscosas o a áreas poco intervenidas.

No obstante, esta observación se debe manejar con mucho cuidado teniendo en cuenta que: 1) se pronosticó equivocadamente que la desaparición de los bosques tropicales y subtropicales llevaría a la desaparición de la leishmaniasis cutánea en Latinoamérica (42), lo cual no ha ocurrido y, por el contrario, ha venido en aumento (43); 2) los muestreos se realizaron en época lluviosa, cuando las poblaciones de estos insectos generalmente son más bajas; 3 ) el número de muestreos en el intradomicilio fue relativamente bajo; 4) la encuesta para evaluar el conocimiento de la enfermedad y del vector se aplicó en una sola vereda.

Además de lo mencionado, la deforestación en el departamento del Guaviare asciende a 6.700 hectáreas/año (informe del convenio SINCHI y la Gobernación del Guaviare, agosto del 2000), circunstancia que en un corto tiempo podría afectar de manera importante la biodiversidad de especies de Lutzomyia y, generar cambios en el comportamiento de estas especies, especialmente en las de mayor tendencia antropofílica, como ha sucedido en otros lugares de Colombia y en algunos países de Latinoamérica. La adaptación a ambientes humanos donde estos insectos tienen fuente de alimento sanguíneo y posibles sitios de cría han convertido la vivienda y las áreas aledañas en zonas de alto riesgo para adquirir la enfermedad $(10,11,44)$.

Desde el punto de vista de salud pública, las especies de los subgéneros Nyssomyia y Psychodopygus, L. flaviscutellata, L. olmeca bicolor, L. antunesi, L. yuilli, y L. panamensis registradas en el presente trabajo, se reconocen por su carácter antropofílico y adaptación al hábitat domiciliar, y por estar comprometidas con la transmisión de parásitos del género Leishmania en diferentes áreas del país y de Latinoamérica $(1,11,16,45$, Vásquez A, González A, Santamaría E, Góngora A, Cabrera OL, Buitrago LE. Detection of Leishmania spp. in canines and species of the genus Lutzomyia in a rural area of Villavicencio, Colombia. XVII American Mosquito Control Association, Orlando, 1 al 5 de abril de 2007). También es importante resaltar que las grandes abundancias para $L$. yuilli registradas en las recolecciones con trampa Shannon, similares a las observadas en otros sitios del país (Grupo de Entomología, datos sin publicarse) $(17,18)$, además de su antropofilia observada en la actividad de picadura con atrayente humano, son comportamientos que favorecerían a corto plazo la entrada de las hembras de estas especies a la vivienda, atraídas por la luz eléctrica (45).

De las especies $L$ hirsuta, $L$. davisi y $L$. carrerai, se conoce su comportamiento antropofílico (1). $L$. hirsutay $L$. davisise han encontrado naturalmente infectadas con Leishmania (Viannia) en Brasil (47) $y$, bajo condiciones experimentales, $L$. carrerai transmitió Leishmania amazonensis a hámster (48). Del subgénero Lutzomyia se registró la presencia de un insecto de L. gomezi, especie importante en salud pública por su compromiso en la transmisión de leishmaniasis en el país $(6,38,49)$.

En relación con la determinación taxonómica de algunas especies en ausencia de machos que no se recolectaron, se aclara que en 20 hembras del subgénero Trichophoromyia y en una del subgenéro Psathyromyia no se definió la especie. Sí fue posible hacerlo con $L$. begonae. L. campbelli y L. sericea.

L. begonae se separó de L. infraspinosa por comparación usando ejemplares de la colección de referencia. Se midieron algunas estructuras de la genitalia y se encontró una diferencia notable con respecto al largo y el ancho de las espermatecas (34). L. campbelli se separó de $L$. dasymera, por el cibario: en L. dasymera, según Llanos (29), el cibario es más pigmentado y presenta un número mayor de dientes verticales (más de 10), mientras que L. campbelli solamente presenta 6; no obstante, el número de dientes 
puede variar. En la espermateca de L. campbelli las estrías que decoran los ductos individuales son nítidas. Estas características fueron confirmadas con las figuras de Young y Duncan (1) y dibujos inéditos de $L$. dasymera depositados en el archivo del Grupo de Entomología del Instituto Nacional de Salud. Aunque la clave de Young y Duncan (1) muestra características en común para $L$. sericea y $L$. andersoni, por distribución geográfica se podría descartar que se trate de esta última especie.

Por otro lado, los resultados de la encuesta aplicada en la vereda Chuapal para determinar el grado de conocimientos por parte de la población sobre la leishmaniasis y sus vectores, indirectamente refuerzan los datos entomológicos y sugieren que en el departamento del Guaviare estos insectos se presentan en densidades muy bajas en ambientes antrópicos y no constituyen una molestia por sus picaduras para la población. Sin embargo, como se mencionó, debido al bajo número de encuestas aplicadas no se puede generalizar este resultado a toda el área de transición Amazonia-Orinoquia, hasta obtener más información al respecto, es decir, extendiendo la aplicación de la encuesta a otras veredas.

En conclusión, se registraron 37 especies de Lutzomyia en el departamento del Guaviare, de las cuales, L. hirsuta, L. yuilli, L. davisi, $L$. carrerai, L. antunesi, L. flaviscutellata y $L$. olmeca bicolor, por su abundancia en el área de estudio, antropofilia y antecedentes vectoriales, podrían estar comprometidas en la transmisión de la leishmaniasis cutánea.

\section{Agradecimientos}

Al Instituto Nacional de Salud y a la Secretaría de Salud del Guaviare, por su apoyo para el desarrollo del trabajo de campo y de laboratorio. A Eunice Aparecida Bianchi Galati de la Universidad São Paulo, Brasil, por su asesoría en la taxonomía del subgénero Sciopemyia.

\section{Conflicto de intereses}

Los autores manifestamos no tener conflictos de intereses en esta publicación.

\section{Financiación}

Instituto Nacional de Salud y Secretaría de Salud del Guaviare.

\section{Referencias}

1. Young DG, Duncan MA. Guide to the identification and geographic distribution of Lutzomyia sand flies in Mexico, the West Indies, Central and South America (Diptera: Psychodidae). Mem Entomol Inst. 1994;54:1-881.

2. Lewis DJ, Young DG, Fairchild GB, Minter DM. Proposals for a stable classification of the phlebotomine sandflies (Diptera: Psychodidae). Syst Entomol. 1977;2:319-32.

3. Tesh RB, Guzmán H. Sand flies and the agents they transmit. In: Beaty BJ, Marquartdt WC, editors. The biology of disease vectors. Niwot: University Press of Colorado; 1996. p. 117-27.

4. Beati L, Cáceres AG, Lee JA, Munstermann LE. Systematic relationships among Lutzomyia sand flies (Diptera: Psychodidae) of Perú and Colombia based on the analysis of $12 \mathrm{~S}$ and $28 \mathrm{~S}$ ribosomal DNA sequences. Int J Parasitol. 2004;34:225-34.

5. Antunes PC. Informe sobre una investigación entomológica realizada en Colombia. Revista de la Facultad de Medicina. 1937;6:3-29.

6. Muñoz G. The sandfly vectors and epidemiology of cutaneous leishmaniasis in the Landázuri focus, Colombia (thesis). London: University of London; 1998. p. 257.

7. Davies $\mathbf{C R}$, Reithinger $\mathbf{R}$, Campbell-Lendrum D, Feliciangeli D, Borges R, Rodriguez N. The epidemiology and control of leishmaniasis in Andean countries.Cad Saude Publica. 2000;16:925-50.

8. Cárdenas $\mathbf{R}$, Pabón $\mathrm{E}$, Anaya $\mathrm{H}$, Sandoval CM. Presencia de Lutzomyia longiflocosa (Diptera: Psychodidae) en el foco de leishmaniasis tegumentaria americana del municipio de Ábrego, Norte de Santander. Primer registro para el departamento. Clon. 2005;3:714.

9. Pardo RH, Cabrera OL, Becerra J, Fuya P, Ferro C. Lutzomyia longiflocosa, posible vector en un foco de leishmaniasis cutánea en la región subandina del departamento del Tolima, Colombia, y el conocimiento que tiene la población sobre este insecto. Biomédica. 2006;26(Supl.1):95-108.

10. Pardo RH. The ecology and control of cutaneous leishmaniasis in the sub-Andean region of South-West Colombia (thesis). Londres: London School of Hygiene and Tropical Medicine; 2006.p. 311.

11. Santamaría E, Ponce N, Zipa Y, Ferro C. Presencia en el peridomicilio de vectores infectados con Leishmania (Viannia) panamensis en dos focos endémicos en el occidente de Boyacá, piedemonte del valle del Magdalena medio, Colombia. Biomédica. 2006;26(Suppl.1):82-94. 
12. Osorno-Mesa E, Morales-Alarcón A, De Osorno F, Ferro-Vela C. Phlebotominae de Colombia (Diptera, Psychodidae) IX. Distribución geográfica de especies de Brumptomyia y Lutzomyia Franca 1924, encontradas en Colombia. Revista Académica Colombiana de Ciencias Exactas, Físicas y Naturales. 1972;14:45-68.

13. Morales A, Minter DM. Estudios sobre flebotominos en Araracuara, Caquetá, Colombia, incluyendo la descripción de Lutzomyia araracuarensis (Diptera: Psychodidae). Biomédica. 1981;1:94-116.

14. Young DG, Morales A. New species and records of phlebotomine sand flies from Colombia (Diptera: Psychodidae). J Med Entomol. 1987;24:651-65.

15. Cipa Group. Computer-aided identification of phlebotomine sandlies of America. (Fecha de consulta: 3 de abril de 2008). Disponible en: http://cipa.snv.jussieu.fr/

16. Montoya-Lerma J, Ferro C. Flebótomos (Diptera: Psychodidae) de Colombia. En: Amat G, Andrade MG, Fernández $F$, editores. Insectos de Colombia. Volumen II. Colección Jorge Álvarez Lleras No. 13. Bogotá D.C.; Academia Colombiana de Ciencias Exactas, Físicas y Naturales; 1999. p. 211-45.

17. Barreto M, Burbano ME, Barreto P. Lutzomyia sand flies (Diptera: Psychodidae) from middle and lower Putumayo department, Colombia, with new records to the country. Mem Inst Oswaldo Cruz. 2000;95:633-9.

18. Wolff M, Sierra D, Murcia LM, Vélez ID. Phlebotominae fauna (Diptera: Psychodidae) in the department of Amazonas, Colombia. Neotrop Entomol. 2003;32:523-6.

19. Molina J, Hildebrand $\mathbf{P}$, Olano V, Muñoz $\mathbf{P}$, Barreto M, Guhl F. Fauna de insectos hematófagos del sur del Parque Nacional Chiribiquete, Caquetá, Colombia. Biomédica. 2000;20:314-26.

20. Barreto M, Burbano ME, Young DG. Description of Lutzomyia (Trichophoromyia) pabloin. sp. and the female of $L$. howardi (Diptera: Psychodidae) from Colombia. J Med Entomol. 2002;39:601-4.

21. Wolff M, Bianchi Galati EA. Description of Pintomyia limafalcaoae and Pintomyia antioquiensis, two new species of phlebotomine sand fly (Diptera, Psychodidae) from the Colombian Andes. Mem Inst Oswaldo Cruz. 2002;97:317-24.

22. Bejarano EE, Duque $\mathbf{P}$, Vélez ID. Taxonomy and distribution of the series pia of the Lutzomyia verrucarum group (Diptera: Psychodidae), with a description of Lutzomyia emberai n. sp. J Med Entomol. 2004;41:83341.

23. Bejarano EE. Lista actualizada de los Psicódidos (Diptera: Psychodidae) de Colombia. Folia Entomol Mex. 2006;45:47-56.

24. Bejarano EE, Duque P, Vélez ID. Redescripción de la hembra de Lutzomyia vattierae (Diptera: Psychodidae, Phlebotominae) de la serranía de La Macarena, Colombia. Biomédica. 2006;26:556-61.
25. Martínez AG. Toda Colombia es mi pasión. Departamento del Guaviare. (Fecha de consulta: 9 de abril de 2008) Disponible en: http://www.todacolombia. com/departamentos/guaviare.html

26. Gobernación de San José del Guaviare. Departamento del Guaviare, el Guaviare en buenas manos. Información general del departamento. (Fecha de consulta: 6 de mayo de 2008). Disponible en:_http://www.guaviare.gov. $\mathrm{co} /$ nuestromunicipio.shtml?apc $=\mathrm{m}-\mathrm{l} 1 \mathrm{-}-\mathrm{\&} \mathrm{m}=\mathrm{f} \& \mathrm{~s}=\mathrm{m}$

27. Instituto Geográfico Agustín Codazzi-IGAC. Diccionario Geográfico de Colombia. Tercera edición. Santafé de Bogotá: Instituto Geográfico Agustín Codazzi; 1996. p. 2504.

28. Young DG. A review of the bloodsucking psychodid flies of Colombia (Diptera: Phlebotominae and Sycoracinae). Technical Bulletin 806. Gainesville: Institute of Food and Agricultural Sciences, University of Florida; 1979. p. 266.

29. Llanos B, Martins AV, Da Silva JE. Estudios sobre os flebotomineos do Peru (Diptera, Psychodidae, Phlebotominae) I- Departamento de Cuzco: 2- Descricao das femeas de Lutzomyia campbelli e Lutzomyia sherlocki e redescricao do macho e descricao da femea de Lutzomyia octavioi. Rev Brasil Biol. 1975;35:655-64.

30. Fairchild GB, Hertig M. Notes on the Phlebotomus of Panamá XVI (Diptera, Psychodidae). Descriptions of new and little-known species from Panamá and Central America. Ann Entomol Soc Amer. 1961;54:237-55.

31. Fairchild GB, Theodor O. On Lutzomyia flaviscutellata (Mangabeira) and L. olmeca (Vargas and Diaz-Najera) (Diptera: Psychodidae). J Med Vet. 1971;8:153-9.

32. Ortíz I, Torres Rojas J. Phlebotomus begonae nov. sp. Nuevo flebotomíneo del subgénero Evandromyia Mangabeira, 1941, en la región sur-occidental amazónica venezolana (Díptera: Psychodidae). Rev Inst Nac Hig. 1975;8:101-5.

33. De Carvalho GM, Falcão AL, Filho JD. Taxonomic revision of phlebotomine sand fly species in the series davisi and panamensis of the subgenus Psychodopygus Mangabeira, 1941 (Diptera:Psychodidae:Phlebotominae). Mem Inst Oswaldo Cruz. 2006;101:129-36.

34. Feliciangeli MD, Ramírez-Pérez J, Ramírez A. The phlebotomine sandflies of Venezuelan Amazonia. Med Vet Entomol. 1988;2:47-65.

35. Ferro C, Morales A. Lista de las especies de flebótomos (Diptera: Psychodidae, Phlebotominae) de Colombia S.A. Biomédica. 1988;8:68-70.

36. Barreto M, Burbano ME, Barreto P. Registros de Lutzomyia (Diptera: Psychodidae) en nuevas localidades de Colombia. Colombia Médica. 2006;37:39-45.

37. Bejarano EE, Duque P, Vélez ID. Estudio de los flebotomineos (Diptera: Psychodidae) antropofílicos de la Serranía de La Macarena, Colombia. Revista Colombiana de Entomología. 2006;32:176-8. 
38. Bejarano EE, Castro $\mathbf{M}$, Pérez-Doria $\mathbf{A}$, HernándezOviedo E, Vélez A, Vélez ID. Primer Informe de Lutzomyia franca en el departamento de Guainía, Amazonia colombiana, y de Brumptomyia mesaiSherlock (Diptera: Psychodidae) en el litoral Caribe colombiano. Neotrop Entomol. 2007;36:990-3.

39. Bejarano EE, Duque P, Vélez ID. Confirmacion de la presencia de Lutzomyia lutziana (Diptera: Psychodidae) en Colombia. Caldasia. 2007;29:153-7.

40. Martins AV. Lutzomyia (Psychodopygus) fairtigi n.sp. from Colombia (Diptera: Psychodidae: Phlebotominae) Proc Entomol Soc Wash. 1970;72:279.

41. Arias JR, Freitas RA. On the vectors of cutaneous leishmaniasis in the Central Amazon of Brazil. 3. Phlebotomine sand fly stratification in a terra firme florest. Acta Amazonica. 1982;12:599-608.

42. Campbell-Lendrum D, Dujardin JP, Martinez E, Feliciangeli MD, Perez JE, Silans LN, et al. Domestic and peridomestic transmission of American cutaneous leishmaniasis: changing epidemiological patterns present new control opportunities. Mem Inst Oswaldo Cruz. 2001; 96:159-62.

43. Zambrano P. Informe de leishmaniasis, Colombia, semanas 1 a 52 de 2005. Inf Quinc Epidemiol Nac. 2006;11:40-3.

44. Vélez ID, Wolff M, Valderrama R, Escobar JP, Osorio L. Community and environmental risk factors associated with cutaneous leishmaniasis in Montebello, Antioquia, Colombia. In: IDRC, editors. Leishmaniasis control strategies: a critical evaluation of IDRC supported research. London: IDRC Publications; 1991. p. 261-74.

45. Young DG, Lawyer PG. New world vectors of the leishmaniases. In: Harrris KF, editor. Current topics in vector research. Vol. 4. New York; Springer Verlag; 1987. p. 29-71.

46. Loiola CF, Da Silva DA, Galati EA. Phlebotomine fauna (Diptera: Psychodidae) and species abundance in an endemic area of American cutaneous leishmaniasis in southeastern Minas Gerais, Brazil. Mem Inst Oswaldo Cruz. 2007;102:581-5.

47. Gil LH, Basano SA, Souza AA, Silva MG, Barata I, Ishikawa EA, et al. Recent observations on the sand fly (Diptera: Psychodidae) fauna of the state of Rondonia, western Amazonia, Brazil: the importance of Psychdopygus davisi as a vector of zoonotic cutaneous leishmaniasis. Mem Inst Oswaldo Cruz. 2003;98:751-5.

48. Ryan L, Lainson R, Shaw JJ, Wallbanks KR. The transmission of suprapylarian Leishmania by the bite of experimentally infected sand flies (Diptera: Psychodidae). Mem Inst Oswaldo Cruz. 1987;82:425-30.

49. Travi BL, Montoya J, Solarte Y, Lozano L, Jaramillo C. Leishmaniasis in Colombia. I. Studies on the phlebotomine fauna associated with endemic foci in the Pacific coast region. Am J Trop Med Hyg.1988;39:261-6. 